\title{
LA CARRERA ARMAMENTISTA: SU SENTIDO COMÚN MATEMÁTICO*
}

\author{
AMIT BHADURI**
}

TODOS CONOCEMOS LA LÓGICA INTUITIVA que subyace a una carrera armamentista. Siempre que dos adversarios tratan de dominar uno al otro a partir de su poderío militar, terminan persiguiéndose mutuamente en la escalada de armamentos. La carrera que resulta - generalmente sustentada en la falsa doctrina de que "sólo es posible negociar desde una posición de fuerza"- es inestable, debido a que ninguna de las partes está dispuesta a llegar a acuerdos, y se apoya, como motivo fundamental en el querer alcanzar la superioridad de su posición militar. Ésta es la razón más sencilla, pero más importante, que está detrás de cualquier carrera armamentista, no importa si es entre dos superpotencias o entre dos potencias regionales.

Para apreciar en términos matemáticos una carrera tan inestable, tómense dos adversarios, cuyos respectivos acervos de armas se miden (unidimensionalmente) por $x$ y y respectivamente. El acervo a

* Traducción de Salvador De Lara R. La versión en inglés de este artículo se publicará en Fesrshright, en honor de R.M. Goodwin.

** Se hace un agradecido reconocimiento a José Alberro por el intercambio de ideas, sin que esto lo involucre en mis errores o puntos de vista. 
que aspira el país $\mathrm{X}$ es $\mathrm{x}^{*} \mathrm{y}$ aquel a que aspira el país $\mathrm{Y}$ es $\mathrm{y}^{*}$. Dada la intensión de dominio militar, el acervo deseado por cada país debe exceder al del adversario. $O$ sea:

$x^{*}=a \cdot y, a>1$
$y^{*}=b \cdot x, b>1$

Cada país construye armamento a una velocidad que depende de la diferencia que percibe entre su acervo real y su acervo deseado, es decir:

$$
\begin{aligned}
& \frac{d x}{d t} \equiv \dot{x}=m\left(x^{*}-x\right), m>0 \\
& \frac{d y}{d t} \equiv \dot{y}=n\left(y^{*}-y\right), n>0
\end{aligned}
$$

Los parámetros $\mathrm{m}$ y $\mathrm{n}$ son la velocidad con que cada país ajusta su acervo real a su acervo deseado. Así, si el país X trata de cubrir en 4 años la diterencia en armamento percibida, $\mathrm{m}=1 / 4$.

Insertando (1) y (2) en (3) y (4) respectivamente, tendremos el siguiente sistema simple de dos ecuaciones diferenciales lineales que deben cumplirse simultáneamente: 1

$$
\left[\begin{array}{l}
\dot{x} \\
\dot{y}
\end{array}\right]=\left[\begin{array}{cc}
-m & m a \\
n b & -n
\end{array}\right] \quad\left[\begin{array}{l}
x \\
y
\end{array}\right]
$$

Es claro que los eigen-valores de la ecuación característica de este sistema, o sea de:

$$
\lambda^{2}+\lambda(m+n)+m n(1-a b)=0,
$$

1 E1 sistema de ecuaciones (5) puede considerarse formalmente similar a las famosas ecuaciones de Richardson (1960). Los términos de "defensa" en Richardson corresponden a nuestros parámetros ma y nb, mientras que su término de "fatiga" tiene aquí los valores de $-\mathrm{m}$ y $-\mathrm{n}$. Nuestra interpretación es distinta de la de Richardson porque nosotros estamos interesados, principalmente, en formalizar el afán de dominio militar. 
tienen partes reales negativas si y sólo si

$$
a b<1
$$

Así, la carrera armamentista descrita en (5) sólo se puede estabilizar en su valor estacionario en el origen $(0,0)$ si $(6)$ se cumple. Sin embargo, esta condición es incompatible con el afán de dominio militar, es decir $a>1$ y b $>1$, tal como lo establecen (1) y (2) respectivamente. Este razonamiento confirma nuestra convicción intuitiva de que una carrera armamentista no puede estabilizarse cuando cada una de las partes trata de dominar militarmente a la otra.

En aras de la exposición, las matemáticas se mantuvieron en los términos más elementales, pero el análisis podría hacerse más elaborado "desplazando" el origen de forma que los valores estacionarios de (5) correspondieran a valores positivos de $\mathrm{x}$ y $\mathrm{y}$. Con ello, un desarmamento parcial sería una posible posición de equilibrio. Sin este cambio (5) especifica que el desarme total es la única posible posición de equilibrio. ${ }^{2}$ Asimismo, las velocidades de ajuste, $\mathrm{m}$ y $\mathrm{n}$, podrían ser variables. Por ejemplo, $m$ o $n$ podrían ser iguales a cero, siempre que no se percibiera una diferencia positiva entre acervo deseado y acervo real, es decir, con $\left(x^{*}-x\right) \leqslant 0$, m sería igual a 0 . Un supuesto así impediría la posibilidad de que la reserva de armas se agotara debido a una política de reposición inadecuada, pero no implicaría un cambio cualitativo en el análisis precedente. ${ }^{3}$ Según las velocidades de ajuste tuvieran valores más altos o más bajos, la carrera armamentista procedería más rápida o más lentamente, pero la propiedad de estabilidad definida en (6) no cambiaría.4 Tampoco habría mayor alteración si no se "linearizara" el afán de dominación y se reemplazara (1) por:

2 Con esto se tendría un sistema de ecuaciones no homogéneas, cuyos términos constantes no homogéneos serían lo que Richardson (1960) considera como el elemento de "agravio".

3 Esta conclusión es evidente a partir del análisis del diagrama de fases que encierra (5) (véase Bhaduri, 1982). Sin embargo, una tasa de obsolescencia muy elevada de los sistemas de armamento -expresión del aspecto tecnológico de la carrera armamentista - justifica la noción de escalada armamentista a una tasa negativa, es decir, desinversión neta en armas. Puede recordarse que una inversión neta negativa como la indicada (en el punto de inversión bruta igual a ce. ro) juega un papel crucial en el "repunte" de varios modelos cíclicos endógenos.

4 Esto es, en la medida en que las velocidades de ajuste $\mathrm{m}$ y $\mathrm{n}$ no son funciones de $\mathrm{x}$ y $\mathrm{y}$, para evitar no linealidades graves. 
$x^{*}=f(y)$, donde $f(y)>y$

$$
\text { y } f>0
$$

En este caso, la condición de estabilidad local es la misma que en (6), aunque el parámetro (a) de (6) tiene que interpretarse como el valor de la derivada de (1a) evaluada en la posición de equilibrio o estacionaria.

Una propiedad importante del modelo sencillo es que logra poner de relieve el papel que juega la inteligencia militar en la carrera armamentista. Por ejemplo, si la inteligencia militar del país X como norma, sobrestima (deliberadamente o no) el acervo de armamento del país $\mathrm{Y}$, dándole una ponderación $\mathrm{K}$ mayor que 1, el acervo de $\mathrm{Y}$ estimado por la inteligencia militar de $\mathrm{X}$ se representará de la siguiente manera:

$\hat{\mathrm{y}}=\mathrm{k} \mathrm{y}$, donde $\mathrm{k}>1$

Por consiguiente, el acervo que $\mathrm{X}$ desea para sí no será el que denota (1), sino:

$x^{*}=a \hat{y}=a k y, a>1$ y $k>1$

Como (1b) sugiere, toda mala información deliberada que conduce a la sobrestimación sistemática de la fuerza militar del adversario no es más que otra forma de desimular el afán flagrante de dominio que pueda tener un país. No es sorprendente que este elemento pueda hacer todavía más inestable la carrera armamentista. 5

Si dominar es el motivo para alcanzar la supremacia militar, la disuasión es su anverso. Un país disuade a su adversario de alcanzar una posición de supremacia militar. Esta doctrina tuvo importancia excepcional en la situación al punto muerto surgida en la posguerra al aparecer las armas termonucleares. Con la posesión del arma nuclear cualquier superpotencia podía infligir un daño avasallador a

5 El instituto de Investigaciones para la Paz Internacional de Estocolmo (Stockholm International Peace Research Institute), en sus Anuarios, ha señalado repetidamente que, al parecer, la $C I A$ tiende sistemáticamente a sobrestimar el gasto militar soviético. Véase, por ejemplo, SIPRI, Yearbook (1981) pp. 8-12. Pardos (1982) hace un recuento detallado de este punto. La condición matemática de estabilidad en presencia de la mencionada sobrestimación sería, $\mathrm{abK}<1$, la cual resulta claramente más restrictiva si $\mathrm{K}>1$. 
indiscriminado a su adversario (civil y militar) incluso en un contrataque (retaliatorio). El daño era tan grande que resultaba insostenible para el que, en potencia, fuera el primer atacante. Así, el arma nuclear daba a un país la capacidad de disuadir a su adversario de obtener una posición de supremacía militar decisiva que le permitiera lanzar cualquier primer golpe ofensivo.

Supongamos que, para tener fuerza de disuasión, el país $\mathrm{X}$ debe alcanzar un poderío militar representado por $\bar{X} .6$ Como éste es un acervo de armamento deseado, pero que no depende del poderío armamentista del adversario, en lugar de (1) tendremos:

$\mathrm{x}^{*}=\overline{\mathrm{x}}$, donde $\overline{\mathrm{x}}$ es una constante positiva arbitraria

De acuerdo con (1c), el país $\mathrm{X}$ sigue una política de disuasión que no está influida por la fuerza militar de su enemigo y, por tanto, no puede verse envuelto en la carrera armamentista. Este proceso es necesariamente estable en el punto en que el país $X$ llega a $\bar{x}$. Esto puede ser formalmente verificado sustituyendo (1) por (1c) y examinando la condición de estabilidad del sistema de ecuaciones diferenciales resultante, o sea (1c) (2) (3) y (4).

Con la sustitución de la ecuación (1) por la (1c) las ecuaciones diferenciales del sistema dejan de ser simultáneas. Éste es el caso lógico extremo en que el país $\mathrm{X}$ actúa militarmente con total independencia del país Y. Por el contrario, la búsqueda de predominio puede verse como un caso de fuerte interacción militar, representada por valores elevados y mayores que uno para los parámetros " $a$ " y " $b$ " en las ecuaciones (1) y (2). Entre estos dos polos hay un amplio espectro de interacción militar débil en el que al menos una de las partes responde de manera relativamente moderada a la escalada militar de su adversario. Esto podría representarse asignándole un valor menor que uno al parámetro "a" o "b" de la ecuación (1) o (2). Dada la condición de estabilidad (6), es evidente que una respuesta moderada por parte de al menos uno de los adversarios aumenta la posibilidad de que la carrera armamentista se estabilice. ${ }^{7}$ Pero en esto mismo radica, también, la tragedia de una carrera armamentista: incluso si el país $\mathrm{X}$ es muy moderado en su respuesta, es decir, el parámetro "a"

$6 \bar{x}$ puede medirse hoy (de manera imperfecta) por el número de proyectiles nucleares. Véase McGuire (1977) para un resumen de este problema de medición.

7 Es decir, es una condición necesaria, pero no suficiente. 
toma un valor muy por debajo de la unidad, un ímpetu de dominación suficientemente fuerte por parte del país $\mathrm{Y}$, representado por un parámetro " $b$ " mucho mayor que la unidad, puede todavía desestabilizar la carrera armamentista al violar la condición (6). En otras palabras, se requiere de ambas partes para estabilizar una carrera armamentista; pero sólo se necesita de una para desestabilizarla, excepto cuando uno de los paises sigue una estrategia independiente de disuasión adecuada [como en (1a)].

Desgraciadamente, la búsqueda de cualquier política independiente de disuación adecuada está limitada por la propia lógica de esta estrategia. La disuasión efectiva exige al país que la implanta el mantenimiento de un nivel inaceptablemente elevado de poder destructivo frente al adversario. En consecuencia, cualquier mejoría incluso de la capacidad de defensa del adversario reduciría la percepción que éste tendría del nivel de riesgo. Esto a su vez socavaría el poder de disuasión. En una situación así, es absurdo pretender distinguir cuáles son los sistemas de armamento "ofensivos" o "defensivos" al desatar una carrera armamentista. Cualquier tipo de sistema armamentista puede desatar la carrera en la medida en que la creciente capacidad "defensiva" de una parte induce a la otra a incrementar su gasto en armas "ofensivas" en su esfuerzo por mantener un poder de disuasión adecuado. Esta confusión de la diferencia entre las armas ofensivas y las defensivas es inherente a la lógica de la disuasión, en la medida en que se debe confiar en el armamento ofensivo para poder desalentar al contrario en su intento de alcanzar objetivos defensivos. ${ }^{8}$

Tómese el caso del país $X$ que quiera mantener una adecuada posición de disuasión mediante el poder ofensivo armado. Asignando el subíndice "o" para denotar fuerza ofensiva, podemos rescribir (1c) como:

$$
\overline{\mathrm{x}}=\overline{\mathrm{x}}_{\mathrm{o}}
$$

Sin embargo, su poder de disuasión puede irse minando paulatinamente a partir de su posición inicial en $\bar{x}_{0}$, debido a la posesión de armamento defensivo por parte de su adversario $\mathrm{Y}$. Así, el acervo de armamento ofensivo deseado por el país $\mathrm{X}$, es decir $\mathrm{x}_{\mathrm{o}}^{*}$, debe ser tal que le permita mantener su poder de disuación en $\bar{x}_{0}$ a pesar de

8 Ésta es la "paradoja de la seguridad": ¡sólo hay mayor seguridad nacional socavando la del adversario! 
la capacidad defensiva de su adversario $Y$. Mediante la utilización del subíndice " $d$ " para denotar poder defensivo, podemos encontrar una sencilla formalización ( $y$ por demás manejable analíticamente) de representar esta situación:

$$
x_{0}=x_{0}^{*}-F\left(y_{d}\right), \quad F^{\prime}>0
$$

o,

$$
\mathrm{x}_{\mathrm{o}}^{*}=\overline{\mathrm{x}}_{\mathrm{o}}+\mathrm{F}\left(\mathrm{y}_{\mathrm{d}}\right), \quad \mathrm{F}^{\prime}>\mathrm{o}
$$

donde $\mathrm{Yd}$ representa el acervo de armas defensivas del país $\mathrm{X}$ y la función " $F$ " representa la manera específica en la que la capacidad defensiva de $\mathrm{Y}$ socava el poder disuasivo de $\mathrm{X}$. Es evidente que (1d) tiene una forma matemática similar a la que tenía (1a), exceptuando el término constante $\overline{\mathrm{x}}_{\mathrm{o}}$, que representa el poder de disuación de $\mathrm{X}$ frente a Y. Por tanto, podemos pensar que el análisis anterior se sostiene: la carrera armamentista se torna inestable si hay fuerte interacción militar entre el armamento ofensivo adquirido por $\mathrm{X}$ (es decir, $\mathrm{x}_{\mathrm{o}}$ ) y el armamento defensivo adquirido por su adversario $\mathrm{Y}$ (es decir, $\left.y_{d}\right)$.

Para desarrollar el análisis, supóngase que el país X se concentra completamente en armas ofensivas siguiendo su estrategia de disuasión, mientras que el país $\mathrm{Y}$ se concentra completamente en armamento defensivo para poder reducir el nivel percibido de amenaza militar. De esta manera, el país Y desearía que su acervo de armas de defensa fuera tal que le permitiera reducir el riesgo militar hasta una constante arbitrariamente baja, $\mathrm{A}$, con lo que $\overline{\mathrm{x}}_{\mathrm{o}}>\mathrm{A}>0$, es decir, ${ }^{9}$

o,

$$
\begin{aligned}
& A=x_{o}-F\left(y_{d}^{*}\right), \bar{x}_{o}>A>0 \\
& y_{d}^{*}=F^{-1}\left(A+x_{o}\right)=G\left(x_{o}\right), G^{\prime}>0
\end{aligned}
$$

en donde, $\mathrm{F}^{-1} \equiv \mathrm{G}$.

Sustituyendo (1d) en (1e) en las relaciones (3) y (4), puede verse que la estabilidad local del sistema simultáneo de ecuaciones diferenciales está dada por la condición,

$$
F^{\prime} G^{\prime}<1
$$

9 Nótese que no puede estimarse que la función "F" pueda ser la misma para los países X y $\mathrm{Y}$ en general en (1d) y (1e) respectivamente. 
donde las derivadas $F^{\prime}$ y $G^{\prime}$ se evalúan en sus valores de equilibrio (suponiendo que existe). De acuerdo con (6) hemos llegado, por tanto, al resultado esperado en (7). Incluso si un pais ( $y$, en este caso), mantiene una posición predominantemente defensiva en cuanto a que fortalece sólo su sistema de defensa, la carrera armamentista puede tornarse inestable si la interacción es fuerte, es decir si se viola la condición (7).10 Vale la pena recordar la razón fundamental de ello: la doctrina de la disuasión genera la "paradoja de la seguridad", o sea el hecho de que un país sólo pueda aumentar su seguridad socavando la de su adversario. En consecuencia, si la situación inicial es, ya, una disuasión basada en la destrucción mutuamente garantizada (MAD*), no tiene mucho sentido establecer la diferencia entre sistemas de armamento "ofensivos" y "defensivos", independientemente de las propiedades materiales de éste, al menos en lo que se refiere a la continuidad de la carrera armamentista. Desde luego, la creciente capacidad marginal de defensa [medida por la derivada de $F$ en (1d)] desestabilizaría aún más la carrera armamentista al romper el equilibrio de disuasión entre dos superpotencias. ${ }^{11}$ En otras palabras, la intensidad de la interacción militar, independientemente de que la respuesta sea con sistemas de armamento "ofensivo" o "defensivo", sigue siendo el determinante crucial de la carrera armamentista cuando la situación inicial está definida por la disuación.

La importancia de la proposición anterior puede apreciarse mejor una vez que desviamos nuestra atención de la carrera armamentista entre superpotencias a la carrera regional. Supongamos que el país $\mathrm{X}$ no sólo tiene armamento convencional, sino que, además, se beneficia de la disuasión nuclear frente a su adversario Y. Sin embargo, esta disuasión nuclear es unilateral, debido a que el país $Y$ no posee ninguna capacidad nuclear; su única manera de disuadir es manteniendo una

10 Si el país $Y$ sólo tiene armas defensivas, no puede, por definición, lanzar ningún ataque. En esta situación, al país $X$ no le interesa una estrategia de disuasión que se base en un segundo ataque. Descartemos este caso extremo, o sea, aquel en que el país $\mathrm{Y}$ no tiene ningún poder ofensivo.

* Esta política de destrucción mutuamente garantizada tiene en inglés las iniciales $M A D$ que significan "loco" o "loca". De ahí que hayamos optado por no poner su equivalente en español. (N. del T.)

11 Esto es claramente importante con relación a la Iniciativa de Defensa Estratégica (Strategic Defense Initiative) popularmente conocida como "Guerra de las Galaxias". Nótese que en nuestra representación matemática, un valor elevado de la derivada de $F^{\prime}$, o sea la eficacia marginal del sistema de defensa, haría que la condición de estabilidad (7) fuera más restrictiva. 
superioridad en lo que se refiere a armas convencionales. 12 La naturaleza de esta disuasión nuclear unilateral de la que se beneficia el país $\mathrm{X}$ se determina mejor a través de su política declarada de renunciar, en todo momento, al primer ataque nuclear. Bajo estas circunstancias, vale la pena investigar si el país $\mathrm{X}$, respaldado por su poder de disuasión nuclear unilateral, se encuentra en posición de ejercer una influencia estabilizadora de la carrera armamentista regional, mediante la reducción del grado de intensidad de la interacción militar. En otras palabras, buscamos las condiciones analíticas bajo las que una disuasión nuclear que beneficia a una sola de las partes pueda tener alguna posibilidad de detener la carrera armamentista regional.

E1 problema puede ponerse en términos más formales mediante la utilización del subíndice "c" y del subíndice " $n$ ", para describir los armamentos convencional y nuclear, respectivamente.

Supóngase que el acervo de armamento convencional deseado por el país X (es decir, $\mathrm{x}_{\mathrm{c}}^{*}$ ) se incrementa conforme el que ya tiene en su poder el adversario $\left(y_{c}\right)$ aumenta, todo ello por medio del proceso de interacción militar habitual descrito desde (1) hasta (1d). Sin embargo, el acervo deseado $x_{c}^{*}$ decrece conforme el país $X$ va poseyendo más armas nucleares $\left(\mathrm{x}_{\mathrm{n}}\right)$, debido en parte, a que, así este país requerirá menos armas convencionales para disuadir a su enemigo de un ataque (es decir, el motivo estratégido de disuasión) y, en parte, a que las restricciones al presupuesto total de defensa reducirían la asignación al programa de armas convencionales dado el desarrollo de un programa más amplio de armamento nuclear. Así, el acervo deseado de armas convencionales del país $\mathrm{X}$ se representará por:

$$
x_{c}^{*}=a y_{c}-h x_{n}, a>o, h>0
$$

donde $\mathrm{x}_{\mathrm{n}}=$ acervo de armas nucleares en posesión del país $\mathrm{X}$. Correspondientemente, el acervo deseado de armamento convencional del país $Y$ (que, supuestamente no tiene armas nucleares) está dado por:

$$
y_{c}^{*}=b x_{c}+p x_{n}, b>1, p>0
$$

Nótese que el país $Y$, normalmente, buscaría mantener superioridad en armamento convencional $(\mathrm{b}>1)$ como única opción estra-

12 Israel frente a sus adversarios del mundo árabe o la India frente a Pakistán son ejemplos de este tipo de disuasión. 
tégica de disuasión frente al adversario. Además, puede pretender incrementar aún más su poderío convencional si su adversario $\mathrm{X}$ incrementa su poderio nuclear [es decir $\mathrm{p}>0$ en (9)].

Describiremos la estrategia de disuasión nuclear unilateral del país $X$ suponiendo que éste incrementa su poderío nuclear siempre que, frente a su adversario, quede rezagado en lo que toca a armamento convencional. En su forma más simple, esto puede representarse por

$$
\dot{x}_{n}=g\left(y_{c}-x_{c}\right), g>0
$$

en donde $\mathrm{g}$ es la velocidad de ajuste del armamento nuclear.

Sustituyendo (8) y (9) en (3) y (4), respectivamente, y utilizando (10) tendremos el siguiente sistema de ecuaciones diferenciales para describir la carrera armamentista regional cuando una sola facción posee armas nucleares:

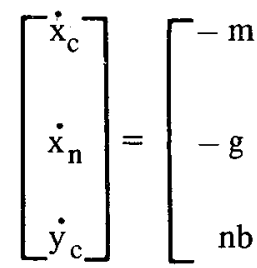

$$
\left.\begin{array}{l}
+m a \\
+g \\
-n
\end{array}\right]\left[\begin{array}{l}
x_{c} \\
x_{n} \\
y_{c}
\end{array}\right]
$$

Al igual que nuestro sistema de ecuaciones (5) (11) se supone (para simplificar) homogéneo. Esto quiere decir que (11) también tiene una solución de equilibrio o estacionaria en el origen $(0,0,0)$ que representa la configuración del desarme total. 13

La ecuación característica de (11) está dada por

$$
\begin{aligned}
& \lambda^{3}+(\mathrm{m}+\mathrm{n}) \lambda^{2}+[\mathrm{mn}(1-\mathrm{ab})-\mathrm{g}(\mathrm{np}+\mathrm{mh})] \lambda+ \\
& \ldots+{ }^{+} \mathrm{mng} \quad(\mathrm{ap}+\mathrm{bh}-\mathrm{p}-\mathrm{h})=0
\end{aligned}
$$

como, según puede verificarse, todos los eigen-valores de la ecuación

13 Véase nota (2) y la correspondiente argumentación. 
(12) tienen términos reales negativos, el origen podría representar una posición (estacionaria) de equilibrio estable si:

Condición $A:(\mathrm{m}+\mathrm{n})>0$, siempre se satisface

Condición $B: \mathrm{mn}(1-\mathrm{ab})>\mathrm{g}(\mathrm{np}+\mathrm{mh})$

Condición $C:(\mathrm{ap}+\mathrm{bh})>(\mathrm{p}+\mathrm{h}) \mathrm{y}$,

Condición D: $\mathrm{mn}(\mathrm{m}+\mathrm{n})(1-\mathrm{ab})>\mathrm{g}[\mathrm{mn}(\mathrm{ap}+\mathrm{bh})-$ $\left.\left(m^{2} h+n^{2} p\right)\right]^{14}$

Nótese que las condiciones $\mathrm{B}$ y $\mathrm{C}$ implican, juntas, que la interacción militar en términos de armamento convencional debe ser débil ( $a b<1$ por condición B), pero, por otro lado, una de las partes debe conducirse a partir de un afán de dominio en términos de armamento convencional ( $\mathrm{a} o$ b debe, necesariamente, ser mayor que la unidad para poder satisfacer la condición C). Esto lo garantiza (9) en donde $b>1$ y la condición (B) sólo puede ser satisfecha si a $<1$ para que $1>$ ab sea posible.

Las condiciones (A) a (D) también establecen que las velocidades de ajuste (parámetros $\mathrm{m}, \mathrm{n}$ y g) son importantes, pues afectan la propiedad de estabilidad en los casos más complejos de la carrera armamentista, en contraste con las condiciones de estabilidad (6) o (7) que son más sencillas. Sin embargo, podemos simplificar considerablemente las condiciones (A) a (D) suponiendo que las velocidades de ajuste son más o menos del mismo orden, es decir,

$$
\mathrm{m}=\mathrm{n}=\mathrm{g}
$$

En este caso especial las condiciones (B) y (D) se simplifican y se convierten, respectivamente, en:

14 La aplicación de las condiciones (A) a (D) se desprende de la utilización del bien conocido teorema de Hurwitz. De acuerdo con él (simplificado para nuestros propósitos), la ecuación característica:

$$
\lambda^{2}+a_{1} \lambda+a_{2}=0
$$

tiene términos reales negativos en sus raíces si $\mathrm{a}_{1}>0$ y $\mathrm{a}_{2}>0$ (véase ecuación (5) y condición (6) ). En el polinomio de tercer órden [tal como en (12)],

$$
\lambda^{3}+a_{1} \lambda^{2}+a_{2} \lambda+a_{3}=0
$$

los términos reales de las raíces son negativos si

$a_{1}>0$ (Condición A), $a_{2}>0$ (Condición $B$ ), $a_{3}>0$ (Condición $C$ ) $y$ $a_{1} a_{2}-a_{3}>0$ (Condición D). 


$$
\begin{aligned}
\text { Condición }(B .1):(1-a b)>(p+h) \\
\text { Condición }(D .1):(1-a b)>(a p+b h)+(p+h)
\end{aligned}
$$

como (D.1) implica (B.1), nos quedan únicamente las condiciones (C) y (D.1) para asegurar que sea estable la carrera armamentista en el caso especial dado por (13).

Algunos ejemplos numéricos muy simples $(a=0.2, b=2.0$, $\mathrm{p}=0.1$ y $\mathrm{h}=0.1$ ) muestran que las condiciones (C) y (D.1) pueden, desde luego, satisfacerse simultáneamente. Por tanto, hay ciertas posibilidades de que una carrera armamentista regional asi se estabilice cuando se da en condiciones de disuasión nuclear unilateral. Una condición necesaria, aunque no suficiente, para ello es que el país que se beneficia de la disuasión nuclear unilateral no insista en tener superioridad o incluso estar a la par en lo que toca a armamento convencional (es decir, $a<1$ mientras $b>1$ ). En general, la interacción militar débil, que implica que todos los términos que son productos cruzados como ab, ap y bh, sean lo bastante pequeños para cumplir con (D.1), se convierte en una posibilidad realizable por parte del país que disfruta de la protección de la disuasión nuclear unilateral. Sin embargo, para hacer real el potencial de estabilización que encierra la carrera armamentista regional, es esencial contar con la sabiduría que reconozca en la moderación y no en la respuesta exagerada la expresión de la auténtica fuerza que está garantizada por la disuasión nuclear unilateral. Es trágico que una política de populismo impida que la fuerza se traduzca en moderación en asuntos militares.

\section{Bibliografía}

BHADURI, A., Domination, Deterrence and Counterforce: an analysis of the strategic objectives and doctrines in the superpower arms race (R.C. Dutt lectures for 1982), Centre for Social Studies, Calcutta (en prensa), 1982.

MCGUIRE, M., "A quantitative study of the strategic arms race in the missiles age", Review of Economics and Statistics, agosto, 1977.

PARDOS, J., The Soviet Estimate: U.S. intelligence analysis and the Russian military strength, Dial, New York, 1982.

RICHARDSON, L.F., Arms and Insecurity, Boxwood, Pittsburgh, 1960.

SIPRI (Stockholm International Peace Research Institute), 1981, anuario. 